\title{
Calidad del Sueño, Estrés Percibido y Desórdenes Temporomandibulares Dolorosos en Adultos Jóvenes de Dos Comunidades Mexicanas
}

\author{
Sleep Quality, Perceived Stress, and Painful Temporomandibular \\ Disorders in Young Adults from two Mexican Communities
}

Celia E. Mendiburu-Zavala; Cesar Pérez-Pérez²; Pedro Lugo-Ancona; Ricardo Peñaloza-Cuevas ${ }^{1}$ \& Enrique Pérez Martínez ${ }^{3}$

\begin{abstract}
MENDIBURU-ZAVALA, C. E.; PÉREZ-PÉREZ, C.; LUGO-ANCONA, P.; PEÑAlOZA-CUEVAS, R. \& PÉREZ, M. E. Calidad del sueño, estrés percibido y desórdenes temporomandibulares dolorosos en adultos jóvenes de dos comunidades mexicanas. Int. J. Odontostomat., 15(4):915-921, 2021.

RESUMEN: Una mala calidad del sueño y estrés, pueden ser la génesis de anomalías temporomandibulares que consisten en una familia heterogénea de desórdenes músculoesqueletales que representan la afección de dolor orofacial crónica más común. El objetivo de este trabajo fue relacionar la calidad del sueño, estrés percibido y desórdenes temporomandibulares dolorosos en adultos jóvenes de dos comunidades mexicanas (Puebla y Yucatán). Material y Métodos: estudio de tipo correlacional, analítico, prospectivo, de corte transversal. Se obtuvo consentimiento informado y voluntario por medio de firmas de 552 sujetos adultos jóvenes: 276 de Puebla $(P), 53 \%(n=147)$ mujeres, $47 \%(n=129)$ hombres, con un promedio de 28 años $( \pm 4,5)$; y 276 sujetos de Yucatán $(Y), 58 \%(n=161)$ mujeres, $42 \%(n=115)$ hombres, con un promedio de 27 años $( \pm 4,9)$. Se les realizó entrevistas con base a tres cuestionarios: Índice de Calidad de Sueño de Pittsburg(ICSP), Escala de Estrés Percibido de Cohen (EEP) e Instrumento de Examinación de Desórdenes Temporomandibulares Doloroso de González (IEDTD). Para el análisis estadístico se utilizó estadística descriptiva (frecuencias y porcentajes), regresión logística y correlación de Pearson. Puebla presenta un porcentaje mayor de individuos con calidad de sueño pobre $83 \%$, comparado con Yucatán, 56 \%. De la misma forma, Puebla muestra un porcentaje mayor de individuos con estrés alto, $82 \%$, comparado con Yucatán, $74 \%$. Por otro lado, Puebla presenta un porcentaje mayor de individuos con DTM dolorosos, 33 \%, comparado con Yucatán, $11 \%$. Asimismo, existe una correlación moderada general entre el estrés percibido y la calidad del sueño en mujeres $(0,335)$ y edades de 30 a 35 años $(0,383)$ ambas con una significancia de $p<0,001$. La calidad de sueño pobre aumenta 4 veces la posibilidad de padecer un DTM doloroso. Vivir en Puebla aumenta hasta 3.1 veces la posibilidad de un DTM doloroso.
\end{abstract}

PALABRAS CLAVE: calidad de sueño, estrés percibido, DTM doloroso.

\section{INTRODUCCIÓN}

Dormir es esencial para la salud física y emocional. El sueño es un estado que ocurre naturalmente de conciencia, actividad sensorial y motora disminuida. Los seres humanos, duermen un promedio de 78 horas en un periodo de 24 horas. Cuando es inadecuado, es un factor de riesgo ya conocido de la diabetes, obesidad, enfermedades cardiacas, depresión, entre otros. Los trastornos del sueño son una amplia categoría de perturbaciones que abarcan todos los tipos de disfunciones que involucran el sueño, incluida la dificultad para conciliarlo por la noche, la mala calidad, la vigilia temprana, las parasomia, los trastornos del movimiento relacionados con el sueño y con los respiratorios. Común en la población gene-

\footnotetext{
${ }^{1}$ Facultad de Odontología de la Universidad Autónoma de Yucatán, México.

${ }^{2}$ Práctica privada

${ }^{3}$ Facultad de Odontología de la Benemérita Universidad Autónoma de Puebla, México.
}

Received: 2021-04-12 Accepted: 2021-07-13 
ral, más de la mitad de los adultos experimenta perturbaciones intermitentes del sueño y entre 15 y $20 \%$ reportan problemas crónicos del mismo. Entre algunas de sus consecuencias, son fatiga durante el día. Los sujetos que sufren de trastornos del sueño, a menudo advierten de una capacidad deteriorada para realizar tareas diarias que involucran memoria, aprendizaje, razonamiento lógico y operaciones matemáticas (Carra et al., 2011; Bassett et al., 2015; Mollayeva et al., 2016).

En consecuencia, una mala conducta del sueño producida en un periodo de tiempo limitado induce cambios en el estado de ánimo y reduce el estado de alerta y rendimiento cognitivo. Estos efectos negativos son acumulativos, específicamente el sueño pobre crónico e insomnio crónico han sido ligados a resultados de salud mental y sicosocial negativos. Existe evidencia, que sugiere que el estrés y sus cambios fisiológicos, son el enlace entre la falta de sueño y sus resultados perjudiciales para la salud. Específicamente, hallazgos de estudios en animales y humanos, apuntan hacia efectos bidireccionales entre el sueño y el eje hipotálamo pituitaria - Adrenal (HPA). La actividad del eje HPA es típicamente evaluada por el ciclo circadiano y su hormona final, cortisol ("hormona del estrés"), además existen múltiples estudios que relacionan el comportamiento del sueño con variaciones en los patrones de secreción diurna de cortisol. Éstos, han mostrado consistentemente, que las disfunciones en el eje HPA tienen un impacto en la salud mental y fisiológica, de esta forma presenta un camino que contribuye a los efectos de la falta de sueño en la salud. De la misma forma, un estudio de privación del sueño en adultos jóvenes sanos realizado por Minkel et al. (2014) demostró que los niveles de cortisol salival seguido de un estresor social, fue significativamente mayor en pacientes con privación del sueño.

Por otro lado, los desórdenes temporomandibulares (DTM) consisten en una familia heterogénea de desórdenes musculoesqueletales que representan la afección de dolor orofacial crónica más común. DTM dolorosos se encuentran asociados con dolor persistente en la región de la articulación temporomandibular (ATM), la región periauricular y los músculos de la cabeza y el cuello. En 2002, la encuesta nacional de entrevista de salud reportó que un $5 \%$ de los adultos presentaban DTM dolorosos (Isong et al., 2008; Maixner et al., 2011).
Por lo tanto, el sueño de los pacientes con DTM dolorosos puede ser usualmente no reparador o de calidad pobre; estas quejas son comúnmente reportadas por la mañana al despertar. Aproximadamente 36 $\%$ de los pacientes con DTM presentan insomnio, y $28 \%$ apnea del sueño. La asociación frecuente entre DTM dolorosos y bruxismo fue revisada recientemente. En un estudio de cohorte de pacientes mujeres con DTM dolorosos y controles libres de dolor que aceptaron dormir en un laboratorio dos noches consecutivas, no se encontró relación entre dolor y el número e intensidad de contracciones musculares mandibulares relacionadas con el bruxismo. Sin embargo, estos mismos pacientes mostraron más esfuerzos respiratorios relacionados con exitaciones (ERA) y un tono muscular mayor en el transcurso de la noche; esto sugiere que los pacientes con DTM pueden encontrarse propensos a mantener un estado de hiperexitación durante la totalidad de la noche demostrando que no pueden "relajarse", al contrario de los pacientes con buena calidad de sueño (Smith et al., 2009; Raphael et al., 2013; Reny \& Klasser, 2018).

Por lo antes mencionado, el objetivo de esta investigación es relacionar la calidad del sueño, estrés percibido y desórdenes temporomandibulares dolorosos en adultos jóvenes de dos comunidades mexicanas: Puebla y Yucatán, durante mayo 2019 a febrero de 2020.

\section{MATERIAL Y MÉTODO}

Tipo de estudio: correlacional, analítico, prospectivo, de corte transversal. Variables de estudio: edad (1835 años); sexo (hombre/mujer); Calidad del sueño (Índice de calidad de sueño de Pittsburgh de Buysse et al., 1989); Estrés percibido (Escala de Estrés Percibido de Cohen et al., 1983); Desórdenes temporomandibulares dolorosos (Instrumento de examinación de desórdenes temporomandibulares dolorosos de Gonzales et al., 2011). Se utilizó la fórmula del tamaño de muestra para poblaciones desconocidas, donde se estimó un tamaño de 276 sujetos por cada comunidad (Puebla y Yucatán). Criterios de inclusión: sujetos que aceptaron participar, de 18 a 35 años y tuvieron una educación mínima de secundaria concluida. Criterios de exclusión: sujetos con enfermedades mentales. De eliminación, una vez iniciados los cuestionarios no desearon seguir realizándolos o no los contestaron como se les solicitó. Tipo de muestreo: no probabilístico, por conveniencia. 
MENDiBURU-ZAVALA, C. E.; PÉREZ-PÉREZ, C.; LUgo-ANCONA, P.; PEÑALOZA-CUEVAS, R. \& PÉREZ, M. E. Calidad del sueño, estrés percibido y desórdenes temporomandibulares dolorosos en adultos jóvenes de dos comunidades mexicanas. Int. J. Odontostomat., 15(4):915-921, 2021.

Se realizó por medio de entrevistas a adultos jóvenes de las ciudades de Mérida, Yucatán y Puebla de Zaragoza, ambas comunidades mexicanas. Se les otorgó una pluma negra o azul. Firmaron una carta de consentimiento informado y voluntario. Seguido, llenaron tres cuestionarios: el primero, fue el Índice de Calidad de Sueño de Pittsburgh (ICSP) (Buysse et al.), en el que se analizó siete componentes diferentes del sueño: calidad del sueño, latencia (tiempo que toma quedarse dormido), duración, eficiencia habitual del sueño, perturbación del sueño, uso de hipnóticos y funcionamiento diurno. Las primeras preguntas analizaron la hora de dormir y despertar usuales del sujeto como también cuánto tardó en quedarse dormido y cuantas horas obtuvo de sueño cada noche. Seguido de esto, se cuantificó eventos sicológicos y físicos específicos como despertar durante la noche, uso del baño durante la noche, no poder respirar fácilmente, ronquidos o tos, si tuvo pesadillas o dolor. De la misma forma, se le preguntó al sujeto, qué tan seguido usó drogas promotoras del sueño, qué tan a menudo le fue complicado permanecer despierto durante actividades diurnas, y qué tanto se le dificultó "mantener entusiasmo para realizar las cosas". Finalmente, el sujeto calificó la calidad del sueño en conjunto en una escala semántica que varía de "muy buena" a "muy mala". Fue una autoevaluación que constó de 19 preguntas autoaplicadas: en la primera sección se encontraron 4 preguntas abiertas y en la segunda, 15 de opción múltiple que fueron rellenadas con una $X$, se les mencionó que solo marcaran una opción, según lo que el sujeto de estudio consideró lo más cercano a su situación (en el último mes). Así, el ICSP incluyó un sistema de puntuación para calcular las 7 puntuaciones secundarias, las cuales van de 0 a 3 . Las puntuaciones secundarias se cuentan, lo que proporciona una puntuación "global" que puede oscilar entre 0 y 21 . Una calificación global de 5 o más indica "calidad de sueño pobre"; mientras más alta la calificación, peor es la calidad del sueño del sujeto. El segundo cuestionario, fue la Escala de Estrés Percibido de Cohen (EEP) (Cohen et al.), instrumento de auto informe que evalúa el nivel de estrés percibido durante el último mes, consta de 14 ítems con un formato de respuesta de una escala de cinco puntos $(0=$ nunca, $1=$ casi nunca, 2 =de vez en cuando, $3=$ a menudo, $4=$ muy a menudo). La puntuación total de la EEP, se obtuvo invirtiendo las puntuaciones de los ítems $4,5,6,7,9$, 10 y 13 (en el sentido siguiente: $0=4,1=3,2=2,3=1$ y $4=0$ ) y sumando entonces las 14 preguntas. La puntuación directa obtenida, indicó que a una mayor puntuación correspondió a un mayor nivel de estrés percibido. Según el puntaje obtenido, se consideró que: de
0-13 existió un bajo estrés, de 14-26 estrés moderado y de $27-40$ estrés alto. El tercer cuestionario, fue el Instrumento de Examinación de Desórdenes Temporomandibulares Dolorosos (Gonzales et al.) (IEDTD). Tuvo una sensibilidad del $98 \%$ y una especificidad de $97 \%$ y ha demostrado identificar con precisión DTM's dolorosos y discriminarlo de otras condiciones que se presentan con síntomas superpuestos. Constó de seis preguntas sobre la duración y tiempo del dolor, así como factores agravantes y de alivio de este. Para calificarlo, se realizó una sumatoria del puntaje de las respuestas. Una respuesta de 'a' se califica con 0 puntos, una respuesta de 'b' con 1 punto y una de 'c' con dos puntos. Una sumatoria mayor o igual a 3 puntos, significó la presencia de un DTM doloroso Para cada cuestionario, se dispuso de 10 minutos.

Pruebas estadísticas. Se utilizó estadística descriptiva e inferencial (Regresión Logística y Correlación de Pearson). Las pruebas estadísticas fueron consideradas significativas cuando $\mathrm{P}<0,05$ y los paquetes estadísticos que se utilizarán fueron el STATGRAPHICS Centurion XVI v. 16.2.04 (StatPoint, 2013) y el SPSS 21 (IBM SPSS, 2012).

\section{RESULTADOS}

Se estudiaron 552 sujetos, 276 de la comunidad de Puebla (EP), $53 \%(n=147)$ mujeres, $47 \%(n=$ $129)$ hombres, con un promedio de 28 años $( \pm 4,5)$. Asimismo, 276 sujetos de la comunidad de Yucatán (EY), $58 \%(n=161)$ mujeres, $42 \%(n=115)$ hombres, con un promedio de 27 años $( \pm 4,9)$.

Comunidad de Puebla, México. De los 276 pobladores estudiados, se encontró una calidad de sueño pobre en el $83 \%(n=229)$ de la población y calidad de sueño normal en el $17 \%(n=47)$. De estos 229 individuos que tuvieron calidad de sueño pobre según edad, los de 18-29 años un $48 \%(n=111)$, y del grupo etario de $30-35$, un $52 \%(n=118)$ respectivamente. De igual manera, según el sexo, la calidad de sueño pobre entre hombres y mujeres fue de $48 \%(n=109)$ y un 52 $\%(n=120)$, respectivamente.

Del mismo modo de los 276 individuos investigados, el $82 \%(n=226)$ de los sujetos presentó un estrés alto, $16 \%(n=44)$ moderado y $2 \%(n=6)$ bajo. De los sujetos con estrés alto $(n=226)$, según la edad, en la escala de $18-29$ años encontramos un $45 \%(n=102)$ y en la de 30-35 años con un porcentaje de $55(n=124)$. 
Asimismo, según el sexo, los hombres, presentaron menor porcentaje de estrés alto, $47 \%(n=107)$, a diferencia de las mujeres, $53 \%(n=119)$.

El $33 \%(n=91)$ presentó DTM dolorosos mientras que el $67 \%(n=185)$ no lo presentó. De los sujetos con DTM doloroso positivo $(n=91)$, el grupo de edad de 18 a 29 años obtuvo un $51 \%(n=46)$ y el grupo de 30 a 35 años obtuvo un $49 \%(n=45)$. El $57 \%(n=52)$ de las mujeres dieron positivo a DTM doloroso mientras que los hombres $43 \%(n=39)$.

Comunidad de Yucatán, México. Se encontró una calidad de sueño pobre en el $56 \%(n=154)$ y calidad de sueño normal en el $44 \%(n=122)$ de la población estudiada. De estos individuos con calidad de sueño pobre $(n=154)$, el grupo de 18-29 tiene un mayor porcentaje con $62 \%(n=96)$ a diferencia del grupo de 30 35 años $38 \%(n=58)$. Según el sexo, los hombres y las mujeres presentaron porcentajes disimiles $41 \%$ $(n=63)$ y $59 \%(n=91)$ respectivamente.

Un $74 \%(n=203)$ presentó un estrés alto, 23 $\%(n=63)$ moderado y $3 \%(n=10)$ bajo. De los sujetos que presentaron estrés alto $(n=203)$, los de 18-29 años, tuvieron con $60 \%(n=122)$ y los de 30-35 años $40 \%(n=81)$. Según el sexo, las mujeres, presentan mayor porcentaje de individuos con estrés alto a di- ferencia de los hombres, $59 \%(n=120)$ y $41 \%(n=$ 83) respectivamente.

El $11 \%(n=31)$ dio positivo a DTM dolorosos, mientras que el $89 \%(n=245)$ dio negativo. De los 31 sujetos con DTM doloroso, el grupo de edad de 18 a 29 años mostro un $55 \%(n=17)$, y el de 30 a 35 años, un $45 \%(n=14)$ y con respecto al sexo, el $65 \%(n=20)$ de las mujeres $y$, los hombres un $35 \%(n=11)$.

Correlaciones entre variables. En general, existe una correlación significativa moderada $(0,316)$ entre la calidad de sueño pobre y el estrés percibido. Por otro lado, en Yucatán existe una correlación baja de 0,163, a diferencia de Puebla, la cual obtuvo una correlación moderada de 0,363 . Se encontró en general, una correlación moderada $(0,3$ a 0,5$)$ en mujeres y en individuos de 30-35 años. En Yucatán, tanto en hombres como en mujeres y en ambos grupos de edad, la correlación fue leve. En Puebla, en hombres se encontró una correlación leve $(0,290)$, mientras que en mujeres moderada $(0,429)$ y en ambos grupos de edad una correlación moderada, $18-29$ años $(0,327)$ y 30 35 años $(0,413)$ (Tabla I).

Para analizar la relación entre la calidad de sueño, estrés percibido, como factor de riesgo para el desarrollo de DTM doloroso, se realizó una re-

Tabla I. Correlación entre la calidad de sueño y estrés percibido en poblanos y yucatecos, según edad y sexo.

\begin{tabular}{lcccccc}
\hline & \multicolumn{2}{c}{ General } & \multicolumn{2}{c}{ Yucatán } & \multicolumn{2}{c}{ Puebla } \\
\hline & Correlación & Sig. & Correlación & Sig. & Correlación & Sig. \\
\hline $\begin{array}{l}\text { General } \\
\begin{array}{l}\text { Sexo } \\
\quad \text { Hombres }\end{array}\end{array}$ & 0,316 & $p<0,001$ & 0,163 & 0,007 & 0,363 & $p<0,001$ \\
$\quad 0,293$ & $p<0,001$ & 0,198 & 0,034 & 0,290 & $p<0,001$ \\
$\quad$ Mujeres & 0,335 & $p<0,001$ & 0,134 & 0,091 & 0,429 & $p<0,001$ \\
Edad & & & & & & \\
$\quad 18-29$ & 0,260 & $p<0,001$ & 0,148 & 0,060 & 0,327 & $p<0,001$ \\
$30-35$ & 0,383 & $p<0,001$ & 0,189 & 0,046 & 0,413 & $p<0,001$ \\
\hline
\end{tabular}

Fuente : instrumentos de medición.

Tabla II. Relación entre calidad de sueño, estrés percibido como factor de riesgo para el desarrollo de DTM doloroso.

\begin{tabular}{|c|c|c|c|c|}
\hline \multirow[t]{2}{*}{ Factor de Riesgo } & \multirow[t]{2}{*}{ Odds ratio } & \multirow[t]{2}{*}{$p$} & \multicolumn{2}{|c|}{ Intervalo de Confianza $95 \%$} \\
\hline & & & Inferior & Superior \\
\hline Constante & 0,069 & 0,005 & 0,0107 & 0,444 \\
\hline Calidad sueño pobre & 4,0258 & $<\_0,001$ & 2,1026 & 7,708 \\
\hline \multicolumn{5}{|l|}{ Estrés percibido (referencia: bajo) } \\
\hline Moderado & 1,0737 & 0,921 & 0,2627 & 4,389 \\
\hline Alto & 0,6945 & 0,599 & 0,1784 & 2,703 \\
\hline Ser hombre & 0,7586 & 0,211 & 0,4918 & 1,17 \\
\hline Edad & 1,0001 & 0,995 & 0,9543 & 1,048 \\
\hline Vivir en Puebla & 3,1952 & $<\_0,001$ & 1,9919 & 5,125 \\
\hline
\end{tabular}

Fuente : instrumentos de medición. 
MENDIBURU-ZAVALA, C. E.; PÉREZ-PÉREZ, C.; LUGO-ANCONA, P.; PEÑALOZA-CUEVAS, R. \& PÉREZ, M. E. Calidad del sueño, estrés percibido y desórdenes temporomandibulares dolorosos en adultos jóvenes de dos comunidades mexicanas. Int. J. Odontostomat., 15(4):915-921, 2021.

gresión logística binaria, que permitieron controlar otras variables como edad, sexo y lugar de residencia. Los resultados del modelo señalaron que fue estadísticamente significativo (X2 = 64,9; gl = 6; $p<0,001)$, por lo que existe al menos una variable que explica el desarrollo de DTM doloroso.

Tal como se observa en la tabla II, los factores de riesgo en el modelo fueron tener una calidad de sueño pobre, y vivir en Puebla. El primero, indica que tener una calidad de sueño pobre aumenta 4 veces más la posibilidad de tener un DTM doloroso. Por su parte, vivir en Puebla, aumenta hasta 3.1 veces la posibilidad de tener un DTM doloroso. Ni el nivel de estrés percibido, sexo o edad fueron factores de riesgo para el desarrollo de DTM doloroso.

\section{DISCUSIÓN Y CONCLUSIONES}

El estudio actual es probablemente el primero en correlacionar la calidad del sueño, estrés percibido y DTM dolorosos en dos poblaciones mexicanas (Puebla y Yucatán). Un mayor porcentaje de individuos poblanos presentaron una calidad de sueño pobre en comparación con los yucatecos, $83 \%(n=229)$ y $56 \%(n=154)$ respectivamente, esto concuerda con los estudios hechos por Mondal et al. (2018) y Tang et al. (2017) los cuales al igual que en la presente investigación, utilizan el ICSP para medir la calidad del sueño en dos poblaciones con diferentes grados de urbanización, en ambos se encuentra, que las ciudades con una mayor población presentaban una menor calidad del sueño.

El porcentaje de individuos con estrés percibido alto fue mayor en Puebla que en Yucatán, 82 $\%(n=226)$ y $74 \%(n=203)$ respectivamente; esto podría deberse a la diferencia poblacional, situación geográfica, usos y costumbres entre las dos comunidades. Un medio urbano con mayor población, estresa de diferentes maneras a los individuos y tiene desiguales consecuencias en su salud física y mental, entre estos estresores se encuentran la contaminación (calidad del aire), violencia e índices delictivos altos (Okkels et al., 2018). El INEGI en su encuesta Nacional de Victimización y Percepción sobre Seguridad Pública (Encuesta Nacional de Victimización y Percepción sobre Seguridad Pública, 2019) en el 2018, que la incidencia delictiva por cada cien mil habitantes en Puebla fue de 29.576, en comparación con Yucatán de 18.635. Es intere- sante mencionar que en Puebla el delito más frecuente es el robo o asalto en calle o transporte público a diferencia del estado de Yucatán en donde este mismo delito es el sexto lugar. Esto demuestra una diferencia significativa entre las dos ciudades. Por otro lado, el Instituto Nacional de Ecología y Cambio Climático (2018), en su informe nacional de la calidad del aire en México 2018, muestra que Puebla no cumple con los límites normados para el contaminante PM2.5 (material particulado en el aire que tiene partículas ultrafinas de un diámetro de 2,5 micrómetros, proveniente de automóviles, camiones, fabricas, quema de madera y otras actividades relacionadas). En Yucatán, debido a que en 2018 no se cumplieron los criterios de suficiencia de información, no fue posible realizar una evaluación.

El porcentaje de individuos con DTM dolorosos fue mayor en Puebla que en Yucatán, $33 \%$ ( $n=$ 91) y $11 \%(n=31)$ respectivamente, no en concordancia con un estudio realizado por Balke et al., (2010) a 223 pacientes localizados en dos ciudades con diferentes grados de urbanización en Irán, concluye que las áreas urbanas sufrían un menor grado de dolor orofacial a diferencia de las rurales $(20,2$ $\%$ vs $46,2 \%$ ). Esto dista del estudio realizado por Goddard \& Karibe (2002) a 192 Nativos Americanos en dos poblaciones con diferentes grados de urbanización, en donde se concluye que el dolor facial es más prevalente en la población más urbanizada que en la rural, $18,6 \%$ y $8,2 \%$ respectivamente, resultados afines con nuestro estudio de México.

Al igual que en Puebla, en Yucatán no se encontró diferencia significativa según la edad y el sexo en calidad del sueño, esto dista del estudio hecho por Fatima et al. (2016) realizado con el ICSP, con una población de 3778 adultos jóvenes, en la que se indica una prevalencia de calidad del sueño pobre más alta en mujeres que en hombres $(65,1 \%$ vs $49,8 \%$ ). Esto puede deberse a que el numero de la población, es mayor.

En Puebla, se encuentra que en el grupo de edad de 30 a 35 años presenta mayor estrés alto a diferencia del grupo de 18-29 años, a diferencia de Yucatán en donde el grupo de 30 a 35 años presenta menor estrés alto que el de 18 a 29. Según el sexo, tanto en Puebla como en Yucatán los porcentajes de mujeres presentan estrés más alto que los hombres. En el estudio realizado a 1640 adultos jóvenes con la EEP de Cohen por Trolle et al. (2017) encuentran que un mayor porcentaje de mujeres te- 
nían estrés alto a comparación de los hombres, coincidiendo con el presente estudio.

En cuanto a la correlación entre la calidad de sueño y estrés percibido en poblanos y yucatecos, según edad y sexo se encuentra que existe una correlación general moderada entre la calidad del sueño y estrés percibido en mujeres $(0,335)$ y especifica en mujeres en Puebla $(0,429)$. Además de una correlación moderada general en el rango de 30 a 35 años $(0,383)$ y especifica en Puebla en los rangos de edades de 18 a 29 años $(0,327)$ y 30 a 35 años $(0,413)$. Un estudio realizado por Charles et al. (2011) en donde analizaron la asociación entre el estrés percibido y la calidad del sueño en 430 oficiales de policía se encuentra que la prevalencia de sueño pobre se incrementaba con niveles altos de estrés percibido. Sin embargo, en este estudio esta tendencia era significante solo en hombres. La diferencia con el presente estudio pudiese radicar en el tipo de población específica a la cual el estudio es dirigido. Por otro lado, un estudio realizado por Roberts et al. (2019) en 335 estudiantes de pregrado con especializaciones en química y biología durante el curso de un semestre encontró una correlación significante entre mayor estrés y menor calidad de sueño en mujeres, pero no en hombres.

Durante la regresión logística, se encuentra que los factores de riesgo a padecer un DTM doloroso en el modelo fue tener una calidad de sueño pobre, y vivir en Puebla. En un estudio realizado por Rener-Sitar et al. (2016) en 609 pacientes diagnosticados con DTM y 88 controles, determinaron que el $90 \%$ de estos tiene una calidad de sueño pobre.

Por todo lo anterior se concluye que, Puebla presenta un porcentaje mayor de individuos con calidad de sueño pobre, y un mayor porcentaje con estrés alto, comparado con Yucatán. Asimismo, presenta un porcentaje mayor de sujetos con DTM dolorosos, en comparación con Yucatán. Por lo tanto, existe una correlación moderada general entre el estrés percibido y la calidad del sueño en mujeres y edades de 30 a 35 años.

La calidad de sueño pobre aumenta 4 veces la posibilidad de padecer un DTM doloroso. Vivir en Puebla aumenta hasta 3,1 veces la posibilidad de un DTM doloroso. Por otro lado, ni el nivel de estrés percibido, sexo o edad fueron factores de riesgo para el desarrollo de DTM doloroso.
MENDIBURU-ZAVALA, C. E.; PÉREZ-PÉREZ, C.; LUGOANCONA, P.; PEÑAlOZA-CUEVAS, R. \& PÉREZ, M. E. Sleep quality, perceived stress, and painful temporomandibular disorders in young adults from two Mexican communities. Int. J. Odontostomat., 15(4):915-921, 2021.

ABSTRACT: Por sleep quality and stress may be the génesis of temporomandibular disorders that consist of a heterogeneous family of musculoskeletal disorders that represent the most common chronic orofacial pain condition. The objective of the study was to relate sleep quality, perceived stress, and painful temporomandibular disorders in Young adults from two Mexican communities (Puebla and Yucatán). a correlational, analytical, prospective, crosssectional study. Informed and voluntary consent was obtained through the signatures of 552 young adult subjects: 276 from Puebla $(P), 53 \%(n=147)$ women, $47 \%(n=129)$ men, with an average of 28 years $( \pm 4.5)$; and 276 subjects from Yucatán $(Y), 58 \%(n=161)$ women, $42 \%(n=115)$ men, with an average age of 27 years $( \pm 4.9)$. Interviews were conducted based on three questionnaires: Pittsburg Sleep Quality Index (ICSP), Cohen's Perceived Stress Scale (EEP) and González's Painful Temporomandibular Disorders Examination Instrument (IEDTD). Descriptive statistics (frequencies and percentages), logistic regression and Pearson's correlation were used for statistical analysis. Puebla has a higher percentage of individuals with por sleep quality, $83 \%$, compared to Yucatán, $56 \%$. In the same way, Puebla shows a higher percentage of individuals with high stress, $82 \%$, compared to Yucatán, $74 \%$. On the other hand, Puebla has a higher percentage of individuals with painful TMD, $33 \%$, compared to Yucatán, $11 \%$. Likewise, there is a general moderate correlation between perceived stress and sleep quality in women (0.335) and ages 30 to 35 years $(0.383)$, both with a significance of $p<.001$. Poor sleep quality increases the chance of painful TMD by 4 times. Living in Puebla increases the possibility of painful TMD up to 3.1 times.

KEY WORDS: sleep quality, perceived stress, painful TMD.

\section{REFERENCIAS BIBLIOGRÁFICAS}

Balke, Z.; Rammelsberg, P.; Leckel, M. \& Schmitter, M. Prevalence of temporomandibular disorders: samples taken from attendees of medital heatlhcare centers in the Islamic Republic of Iran. J. Orofac. Pain, 24(4):361-6, 2010.

Bassett, S. M.; Lupis, S. B.; Gianferante, D.; Rohleder, N. \& Wolf, J. M. Sleep quality but not sleep quantity effects on cortisol responses to acute psychosocial stress. Stress, 18(6):638-44, 2015.

Buysse, D. J.; Reynolds, C. F.; Monk, T. H.; Berman, S. R. \& Kupfer, D. J. The Pittsburgh Sleep Quality Index: a new instrument for psychiatric practice and research. Psychiatry Res., 28(2):193213, 1989. 
Carra, M. C.; Huynh, N.; Morton, P.; Rompré, P. H.; Papadakis, A.; Remise, C. \& Lavigne, G. J. Prevalence and risk factors of sleep bruxism and wake-time tooth clenching in a 7- to 17-yr-old population. Eur J. Oral Sci, 119(5):386-94, 2011.

Charles, L. E.; Slaven, J. E.; Mnatsakanova, A.; Ma, C.; Violanti, J. M.; Fekedulegn, D.; Andrew, M. E.; Vila, B. J. \& Burchfiel, C. M. Association of perceived stress with sleep duration and sleep quality in police officers. Int. J. Emerg. Ment. Health, 13(4):22941, 2011.

Cohen, S.; Karmack, T. \& Mermelstein, R. A global measure of percibed stress. J. Health Soc. Behav., 24(4):385-96, 1983.

Encuesta Nacional de Victimización y Percepción sobre Seguridad Pública (ENVIPE), 2019. Disponible en: https://www.inegi.org.mx/ programas/envipe/2019/19

Fatima, Y.; Doi, S. A. R.; Najman, J. M.; \& Al Mamun, A. Exploring gender difference in sleep quality of young adults: Findings from a large population study. Clin. Med. Res., 14(3-4):138-44, 2016.

Goddard, G. \& Karibe, H. TMD prevalence in rural and urban Native American populations. Cranio, 20(2):125-8, 2002.

Gonzales, Y. M.; Schiffman, E.; Gordon, S. M. \& Seago, B. Development of a brief and effective temporomandibular disorder pain screening questionnaire. J. Am. Dent. Assoc., 142(10):118391, 2011.

Instituto Nacional de Ecología y Cambio Climático (INECC). Mexico ante el cambio clímatico. Ciudad de México, Gobierno de México, 2018. Disponible en: https://www.gob.mx/inecc

Isong, U.; Gansky, S. A. \& Plesh, O. Temporomandibular joint and muscle disorder-type pain in U.S. adults: the National Health Interview Survey. J. Orofac. Pain, 22(4):317-22, 2008.

Maixner, W.; Diatchenko, L.; Dubner, R.; Fillingim, R. B.; Greenspan, J. D.; Knott, C.; Ohrbach, R.; Weir, B. \& Slade, G. D. Orofacial Pain Prospective Evaluation and RiskAssessment Study - The OPPERA Study. J. Pain, 12(11):T4-T11.e2, 2011.

Minkel, J.; Moreta, M.; Muto, J.; Htaik, O.; Jones, C.; Basner, M. \& Dinger, D. Sleep deprivation potentiates HPA axis stress reactivity in healthy adults. Health Psychol., 33(11):1430-4, 2014.

Mollayeva, T.; Thurairajah, P.; Burton, K.; Mollayeva, S.; Shapiro, C. M. \& Colantonio, A. The Pittsburgh sleep quality index as a screening tool for sleep dysfunction in clinical and non-clinical samples: A systematic review and meta-analysis. Sleep Med. Rev., 25:52-73, 2016.

Mondal, H.; Mondal, S. \& Baidya, C. Comparison of perceived sleep quality among urban and rural adult population by Bengali Pittsburgh Sleep Quality Index. Adv. Hum. Biol., 8(1):36-40, 2018.

Okkels, N.; Kristiansen, C. B.; Munk-Jørgensen, P. \& Sartorius, N. Urban mental health: Challenges and perspectives. Curr. Opin. Psychiatry, 31(3):258-64, 2018.

Raphael, K. G.; Janal, M. N.; Sirois, D. A.; Dubrovsky, B.; Wigren, P. E.; Klausner, J. J.; Krieger, A. C. \& Lavigne, G. J. Masticatory muscle sleep background electromyographic activity is elevated in myofascial temporomandibular disorder patients. J. Oral Rehabil., 40(12):883-91, 2013.

Rener-Sitar, K.; John, M. T.; Pusalavidyasagar, S. S.; Bandyopadhyay, D. \& Schiffman, E. L. Sleep quality in temporomandibular disorder cases. Sleep Med, 25:105-12, 2016.

Reny, L. \& Klasser, G. Orofacial Pain: Guidelines for Assessment, Diagnosis, and Management. 5a ed. Batavia, Quintessence, 2018.

Roberts, L.; Gao, C. \& Scullin, M. Sex Differences in the Effects of Perceived Stress on Sleep Quality: A Structural Equation Model Approach. Sleep, 42(1):A113, 2019.

Smith, M. T.; Wickwire, E. M.; Grace, E. G.; Edwards, R. R.; Buenaver, L. F.; Peterson, S.; Klick, B. \& Haythornthwaite, J. A. Sleep Disorders and their Association with Laboratory Pain Sensitivity in Temporomandibular Joint Disorder. Sleep, 32(6):779-90, 2009.
Tang, J.; Liao, Y.; Kelly, B. C.; Xie, L.; Xiang, Y. T.; Qi, C.; Pan, C.; Hao, W.; Liu, T.; Zhan, F. \& Chen, X. Gender and Regional Differences in Sleep Quality and Insomnia: A General Populationbased Study in Hunan Province of China. Sci. Rep., 7:43690, 2017.

Trolle, N.; Lund, T.; Winding, T. N. \& Labriola, M. Perceived stress among 20-21 year-olds and their future labour market participation - An eight-year follow-up study. BMC Public Health, 17(287):1-9, 2017.

\section{Dirección de correspondencia}

Dra. Celia Elena del Perpetuo Socorro Mendiburu-Zavala

Facultad de Odontología

Universidad Autónoma

Yucatán

MÉXICO

\section{ORCID 0000-0001-9397-1137}

\author{
E-mail: cel_mendi@hotmail.com \\ mzavala@correo.uady.mx
}

